\title{
THE TRANSITION THROUGH RESONANCE OF A NONLINEAR NON-AUTONOMOUS SYSTEM
}

\author{
P. B. CHAPMAN ${ }^{1}$ \\ (Received 14 October 1989; revised 30 April 1991)

\begin{abstract}
An approximate nonlinear perturbation analysis for the re-entry roll resonance model is given. The results are used to identify the dynamic processes involved, as characterised by terms in the model equations, and to suggest a prudent management rule for this and similar transiently-resonant systems.
\end{abstract}

\section{Introduction}

The presence of non-autonomous terms in nonlinear systems may complicate their analysis. Direction-field singularities are no longer available as a guide to it, since the related autonomous system has always one member

$$
\frac{d x_{n+1}}{d t}=1
$$

with nowhere-vanishing right-hand side. The potential for complication is not reduced if the system under consideration is oscillatory and undergoes transient or persistent resonance. But compensation for the absence of direction field singularities may occur if there are families of quasi-steady solutions, or of asymptotic solutions, available as the bases for perturbation analysis of the system. Thus the main object of the further investigation below of a previously-studied non-autonomous system is the perturbation of a family of its quasi-steady solutions. Calculations of this kind are useful in identifying the essential processes which determine the dynamics in physical problems, and the essential variable scales. They may also produce simple

\footnotetext{
'Department of Mathematics, The University of Western Australia, Nedlands, W.A., 6009.

(C) Copyright Australian Mathematical Society 1992, Serial-fee code 0334-2700/92
} 
rules-of-thumb for the design or management of systems with similar underlying mechanics.

For a problem describing an externally axi-symmetric projectile with displaced mass center which undergoes re-entry roll resonance, Kevorkian [5], [6] developed a plausible, non-autonomous analytical paradigm in essentially two physical variables-spin about the symmetry axis and a component of the precessional pitch/yaw motion of the symmetry axis-which, as will be seen, has two families of quasi-steady solutions. He used a formal mathematical procedure to select and perturb one of these families. The results were then used to confirm, among other things, apparently-divergent ("sustained resonant") solutions observed in some numerical integrations of the full equations, but little physical interpretation was advanced for the unusual phenomenon described. Granted, intuition is a chancy guide to rigidbody dynamics with even small aerodynamic inputs, but there is some common experience available in special case of axi-symmetric bodies-and of course, there are the special solutions of classical mechanics to the zeromoment problem. For example, even occasional viewers of American Football's minute television coverage would be struck by the apparent stability afforded the ellipsoidal ball (whose mass center is likely to be slightly displaced) by the quarterback's imparting spin to it, and the classical solutions describe a continuum of angular-momentum-conserving modes at constant energy, in which the motion to a fixed observer is precession of, and spin about, the symmetry axis of the axi-symmetric body.

Thus Kevorkian's “distinguished solution" leading to sustained resonance seemed remarkable, as it is available no matter how small the magnitude of (what can be interpreted as) an initial pitch/yaw disturbance $\rho$ for sufficiently large spin $p_{0}$; unavailable for small enough spin no matter how large the initial pitch/yaw disturbance; with both extreme cases independent of the size of a coupling parameter $\varepsilon$ ([5], Section 4, paragraph whose first equation is 4.12 ).

The nature of the sustained resonance is even more striking. Kevorkian's analysis predicted a divergence (the present usage is the engineering one: uncomfortably large, and growing) in spin and axial precession rates, while latter's amplitude $\rho$ remains locally almost constant. These observations notwithstanding, a sustained resonant solution family exists, but it will be shown to be unavailable to a perturbation theory, being generated by conditions near the left hand limit outside the range of perturbations. The family is in fact characterised by a monotone slow decay in the pitch/yaw amplitude as the time-like variable increases (Section 2 below) and so describes recovery from certain large pitch/yaw disturbances through a spin divergence.

Thus it seemed to the writer that either the paradigm used as the basis of the description was inappropriate, or that the analysis in [5] was deficient. 
Consideration of the first alternative suggested the paradigm to be a notunreasonable reduction of the full equations; but the analysis given in [5] is in error. In brief this is: a matching procedure between inner and outer solutions is spurious because the inner solution's interval of validity does not intersect that of the outer solution. A correction was attempted by Kath [3], [4] in a more general context; there is no apparent modification in a later study [6] by Kevorkian. A discussion of these assertions is given below in Section 5 .

A correct approximate analysis of Kevorkian's paradigm is advanced in Sections 2, 3 here, with a proof of its validity in Section 4. The key to the successful local application of a modification of the averaging algorithm (for an account of the standard version, see Guckenheimer and Holmes [2]) is a preliminary transformation of the independent variable, along the lines of Lighthill's [7] technique for "rendering solutions ... uniformly valid", which here extends the interval of applicability of locally-valid averaged solutions beyond that which would otherwise be available to them. A justification of the non-standard averaging is given in Section 4. These local approximations (Section 3) are then matched to solution approximations which apply in a region where the dynamics are not resonant. The latter class (also used in [5]) are stated in Section 2.

The approximate solutions obtained in Section 3 have properties different from those of the solutions advanced in [5]. The essential process involved can be interpreted as an energy exchange, determined by average rates, between the spin and pitch/yaw modes of the projectile due to weak coupling at a transient resonance, with both the amplitude and the frequency of the pitch/yaw mode affected. Energy is not, however, generally conserved in the exchange-presumably a balance could be struck through some other coupling with the projectile's translational energy. The sense of the transfer is determined essentially by a relative phase which cannot be inferred at " $-\infty$ ", but the sense is from spin to pitch/yaw for almost half the $2 \pi$ range of the relative phase, and the reverse for almost the remainder. Away from the interaction, all solutions decay with time in pitch/yaw, but the energy exchange process implies that the pitch/yaw amplitude of some solutions has a maximum near the resonant transition. In a non-perturbation theory, sufficiently large pitch/yaw displacements might lead either to aerodynamic inputs beyond the scope of the present model (and hence to other divergence problems), or to eventual recovery in pitch/yaw through the sustained resonant spin divergence.

The phenomenon of sustained resonance is addressed, as already noted. In Section 2 this class of solutions is identified on an interval which includes the origin, but not $-\infty$. However, these solutions turn out to be incompatible 
with a complete set of perturbation solutions whose domain includes that limit, so the conclusion is that, at least for sufficiently-small values of the controlling small parameter, sustained resonance does not occur for accessible conditions at $-\infty$. This conclusion seems to be in agreement with numerical simulations of a more elaborate problem reported in [5].

Finally, a suggestion is made for the reduction of resonant intersection on the basis of the paradigm.

\section{Quasi-steady and some local asymptotic solutions}

The equations of Kevorkian's paradigm are [5], 2.14a, b, $c$ and 2.11e. The proliferation of symbols in its analysis is much reduced if the problem is restated as follows. Choose a new time $t$ origin to be the first point at which $\omega$ and $p$ are equal, to $K$ say. Such a point will almost certainly exist, on account of the monotone behaviour of $\omega$, and the structure of the equations. Next, make the scaling changes

$$
\begin{aligned}
t & =t_{N}(K \sqrt{2})^{-1}, \quad x=x_{N} K^{-1}, \quad p=p_{N} K, \\
\psi & =\psi_{N}, \quad \varepsilon=\varepsilon_{N} K \sqrt{2}
\end{aligned}
$$

in Kevorkian's equations $(2.11 \mathrm{e}, 2.14 \mathrm{a}, \mathrm{b}, \mathrm{c}$ ) to obtain the system to be used below which, after dropping the now superfluous suffix, is

$$
\begin{gathered}
\frac{d^{2} x}{d t^{2}}+\frac{1}{2}\left(p^{2}+\omega^{2}\right) x=0, \\
\frac{d p}{d t}=\varepsilon x \sin (\psi) \omega^{2} \\
\frac{d \psi}{d t}=p \\
\omega^{2}(\varepsilon t)=\exp (\varepsilon t) .
\end{gathered}
$$

Since, as it turns out, integration of (2.4) leads to an integral which diverges at $-\infty$, there can be no sensible initial-value problem requiring a $\psi$ value at that limit. Thus the system (2.2-5) is first regarded as an initial-value problem to be integrated in both senses from the $t$ origin, where one initial condition is

$$
p(0)=1 ;
$$

the others are parameters of the problem and assumed to be of unit order. Here it is noted that, although the coefficient $\varepsilon$ in (2.3) could be removed by a further scaling of the variable $x$, it is better to take (2.2-6) as perturbation equations of the underlying system on a particular $x$ scale. 
While this approach to the problem-essentially the examination of all combinations of initial states and non-homogeneous term which lead to the same spin at the first instant of resonance-simplifies the presentation of the analysis, it will make the interpretation of the results more complicated. However, it seems to the writer that, on balance, there are advantages in this approach. Consideration of the physical initial-value problem from the left-hand $(-\infty)$ limit will be given later.

There are two families of quasi-steady solutions of the system (2.2-5) which could be perturbed. The first of these

$$
p=0 ; \quad \psi= \pm m \pi, \quad m=0,1,2, \ldots,
$$

and $x$ any solution of the equation

$$
\frac{d^{2} x}{d t^{2}}+\frac{1}{2} \omega^{2} x=0
$$

is not immediately relevant to the present study, as the condition (2.6) is not met. However, its perturbations could conceivably represent an asymptotic state of the system (2.2-5) in the limit $t \rightarrow \infty$, even for small $\varepsilon$. This is not the case, for, with $m=0$ in (2.7) as an example, on introducing a new variable

$$
\varepsilon t=u,
$$

and using the Liouville-Green [8] approximation, it is found that a particular asymptotic solution of $(2.8)$ is

$$
x \simeq-\frac{\varepsilon A}{4} \omega^{-1 / 2} \sin (\omega / \sqrt{2} \varepsilon)
$$

(again, for example) with $A$ a constant. It follows that the perturbation equation governing $\psi$ is

$$
\frac{d^{2} \psi}{d \omega^{2}}+\frac{1}{\omega} \frac{d \psi}{d \omega}+A \omega^{-1 / 2} \sin (\omega / \sqrt{2} \varepsilon) \psi=0
$$

This linear equation may be shown to have only one bounded solution family for fixed $\varepsilon$ and $\omega \rightarrow \infty$

$$
\psi \simeq C\left(1+2 \varepsilon^{2} A \omega^{-1 / 2} \sin \left(\frac{\omega}{\varepsilon \sqrt{2}}-2 \varepsilon^{2} A \omega^{-1 / 2} \cos \left(\frac{\omega}{\varepsilon \sqrt{2}}\right)\right)\right)
$$

where $C$ is an arbitrary constant. Then $p$ may be calculated from the version of $(2.4)$

$$
\frac{d \psi}{d \omega}=\frac{2 p}{\varepsilon \omega}
$$

and this calculation shows that the perturbed solution $p$ has oscillatory growth as $\omega \rightarrow \infty$, so that the solution (2.7-8) is unstable, and unsuitable as a limiting form. 
A second family of quasi-steady solutions of $(2.2-6)$ is

$$
x=0, \quad p=1, \quad \psi=t+\psi(0),
$$

and perturbations of this family are the "inner solutions" in Section 3 below.

There are also approximations to solutions of $(2.2-5)$ which are asymptotic in $\varepsilon$ and apply on intervals of extent $\sim O\left(\varepsilon^{-1}\right)$ on either side of, but excluding $O\left(\varepsilon^{-1 / 2}\right)$ sub-intervals containing, the origin. They are conveniently called "outer solutions", and their limit form for small values of the natural variable $(\varepsilon t)$ will be used to "match" solution approximations-the inner solutions-which apply in the sub-intervals containing the origin.

These outer solutions are correctly identified by Kevorkian ([5], equations $3.24 a, b ; 3.25 a)$ and in the present notation they are

$$
x(t) \simeq 2 \operatorname{Re}\left\{A_{ \pm}\left(\frac{p_{ \pm}^{2}+\omega^{2}}{2}\right)^{-1 / 4} \exp \left( \pm i \int_{0}^{\varepsilon t}\left(p_{ \pm}^{2}+\omega^{2}(u)\right)^{1 / 2} d u /(\varepsilon \sqrt{2})\right)\right\}
$$

$$
\begin{array}{cc}
\left.\begin{array}{rl}
p \simeq p_{+}, \quad t>0 \\
\simeq p_{-}, \quad t<0
\end{array}\right\} & \begin{array}{l}
p_{+}, p_{-} \text {constants } ; \\
\left|p_{+}-1\right|,\left|p_{-}-1\right| \sim O\left(\varepsilon^{1 / 2}\right)
\end{array} \\
\psi \simeq p_{ \pm} t+\psi_{1 \pm} ; & \psi_{1+}, \psi_{1-} \text { constants }
\end{array}
$$

In (2.15) the constants $A_{+}$and $A_{-}$are complex; the apparently artificial complex notation is introduced here for later convenience.

A heuristic description of approximations to a special family of solutions of $(2.2-6)$ - the sustained resonant [5] family-is now given. This approximation is used primarily for $\varepsilon t>0$, although it continues back to $\varepsilon t<0$; and it applies for small $\varepsilon$ but not, as it turns out, for unit order disturbances in the $x$ variable. It is found by picking a particular family of LiouvilleGreen approximations (Olver [8]) to the solution of (2.2) on the assumption that $p$ and its derivative with respect to $\omega$ are respectively approximated on a useful $\varepsilon t$ interval as

$$
p \approx \omega, \quad \frac{d p}{d \omega} \approx 1,
$$

so that $p$ is slowly varying. These approximations (2.18) are suggested by some of the numerical simulations reported by Kevorkian ([5], Figure 2). The solution family foreshadowed above, and the $p$ approximation (2.18), must then be demonstrated consistent with (2.2-4) by using them to show that an improved approximation to $p$ is a perturbation. 
The only approximations to solutions of (2.2) consistent with $(2.3-4,2.18)$ will be (in real variable form), some of the family

$$
\begin{aligned}
x & \simeq A \omega^{-1 / 2} \cos \left(\int_{0}^{\varepsilon t}\left(p^{2}+\omega^{2}(u)\right)^{1 / 2} d u / \varepsilon \sqrt{2}+\psi(0)\right) \\
& \simeq A \omega^{-1 / 2} \cos \left(\int_{0}^{\varepsilon t}\left(p+\frac{\omega-p}{2}\right) d u / \varepsilon+\psi(0)\right),
\end{aligned}
$$

where the tangent plane at $p=\omega$ is used to approximate the cone

$$
\mathfrak{I}=\left(p^{2}+\omega^{2}\right)^{1 / 2} / \sqrt{2}
$$

in the integrand. (All other Liouville-Green approximations

$$
x \simeq A \omega^{-1 / 2} \sin ((2 \omega / \varepsilon)+h), \quad h \text { constant }
$$

to the solution of (2.2) constructed on the basis of (2.18) resonate when they, and the similarly approximated solution of $(2.4), \psi \simeq(2 \omega / \varepsilon)+\psi(0)$, are substituted in (2.3) to calculate the correction to (2.18): averaging arguments can there be used to reduce (2.3) to

$$
\begin{aligned}
\frac{d p}{d \omega}= & 2 \omega x \sin (\psi) \simeq 2 A \omega^{1 / 2} \cos (\psi(0)-h) \\
& + \text { rapidly oscillating terms, neglected on average. }
\end{aligned}
$$

So the implied correction to $p$ is proportional to $\omega^{3 / 2}$, and not a perturbation of the approximation (2.18) used to generate it.) The expression (2.19), if justified by its consistency, shows the characteristic monotone decay in amplitude.

Since $p$ is assumed to be slowly varying, the integral of $(2.4)$ is expressed as

$$
\psi \simeq \int_{0}^{e t} p(u) d u / \varepsilon+\psi(0)
$$

Equation (2.3) then becomes, on using the approximations (2.18-19), taking for example the case $\psi(0)=0$, and with $\omega=\exp (\varepsilon t / 2)=\exp (u / 2)$

$$
\begin{aligned}
\frac{d p}{d \omega} & \simeq A \omega^{1 / 2}\left(\sin \left(\int_{1}^{\omega} \frac{p-\omega}{\omega} d \omega / \varepsilon\right)+\sin (4(\omega-1) / \varepsilon)\right) \\
& \simeq \mathrm{A} \omega^{1 / 2}\left(\int_{1}^{u} \frac{p-\omega}{\omega} d \omega+\varepsilon \sin (4(\omega-1) / \varepsilon)\right) .
\end{aligned}
$$

Here a new constant $\mathbf{A}=A / \varepsilon$ is introduced, and the assumption is made which is central to consistency, namely: that the argument of the sine remains small on the interval. This is the only way to generate solutions $p$ satisfying the empirical conditions (2.18). Now substitute in (2.21)

$$
V=\int_{1}^{\omega} \frac{p(\omega)-\omega}{\omega} d \omega
$$


to obtain from it the linear, non-homogeneous equation

$$
\frac{d^{2} V}{d \omega^{2}}+\frac{1}{\omega} \frac{d V}{d \omega}-\mathbf{A} \omega^{-1 / 2} V=-\omega^{-1}+\varepsilon \mathbf{A} \omega^{-1 / 2} \sin (4(\omega-1) / \varepsilon) .
$$

The existence theory for the homogeneous version of (2.23) shows asymptotic stability only if the constant $A$ is negative, as is now assumed, in which case a solution pair can be asymptotically approximated as $\omega \rightarrow \infty$ from expressions

$$
\begin{aligned}
V_{\mathrm{CF}} & =\omega^{-3 / 8} \exp \left( \pm i b \omega^{3 / 4}\right)\left(1+O\left(|\mathbf{A}|^{-1 / 2} \omega^{-3 / 4}\right)\right), \\
\frac{d V_{\mathrm{CF}}}{d \omega} & = \pm 3 i b \omega^{-5 / 8} \exp \left( \pm i b \omega^{3 / 4}\right)\left(1+O\left(|\mathbf{A}|^{-1 / 2} \omega^{-3 / 4}\right)\right) / 4, \\
b & =4|\mathbf{A}|^{1 / 2} / 3 .
\end{aligned}
$$

If $|A|$ is large, these approximations will certainly be valid on intervals $1<$ $\omega<\infty$. Particular integrals for the separate parts of the right hand side of (2.23) have combined asymptotic representation as $\omega \rightarrow \infty$

$$
V_{P I} \simeq-|\mathbf{A}|^{-1} \omega^{-1 / 2}+\varepsilon^{3}|\mathbf{A} / 16| \omega^{-1 / 2} \sin (4(\omega-1) / \varepsilon),
$$

with the natural representation for the derivative and, if $|\mathbf{A}|$ is not too large, then these will apply on the same $\omega$ interval as the complementary functions (2.24).

In order for the reduction leading to the approximate equation (2.21) to be consistent, it is necessary that both $|V / \varepsilon|$ and $|d V / d \omega|$ remain uniformly small on the interval of interest-that is (for the present), $\omega>1$. The latter condition follows from the definition (2.22), which requires

$$
p=\omega\left(1+\frac{d V}{d \omega}\right) \text {. }
$$

The initial conditions on (2.23) are

$$
V(1)=0, \quad \frac{d V}{d \omega}(1)=0
$$

as follow from the definition (2.22) of $V$ and the condition (2.6); here

$$
p(\omega=1)=1 \text {. }
$$

Thus the terms dominating the approximation to the solution of (2.23) are

$$
V \simeq|\mathbf{A}|^{-1}\left(\omega^{-3 / 8} \cos \left(b\left(\omega^{3 / 4}-1\right)\right)-\omega^{-1 / 2}\right),
$$

(which implies the improved approximation

$$
\left.p \simeq \omega\left(1+O\left(|\mathbf{A}|^{-1 / 2} \omega^{-5 / 8}\right)\right)\right)
$$

if the small correction required for the derivative condition (2.26) and other 
relatively small terms are ignored. So the condition for consistency $-|V / \varepsilon|$ small-necessitates $|\mathbf{A} \varepsilon|^{-1}=|A|^{-1}$ small, that is to say the amplitude in pitch/yaw (2.19) is large

$$
|A| \gg 1 \text {. }
$$

When this inequality is satisfied the derivation of the sustained resonant solution shown above is consistent, but it is incompatible with the assumption underlying the scaling (2.1) that the disturbances are unit order.

The limit initial forms on $x$ and $\psi$ which augment the specification of the sustained resonant solutions, obtained from the limit forms of approximations (2.19-20) are

$$
\begin{aligned}
& \psi(\varepsilon t \rightarrow 0) \simeq t+\psi(0), \\
& x(\varepsilon t \rightarrow 0) \simeq A \cos (t+\psi(0)), \quad A<0,
\end{aligned}
$$

and

$$
p(\varepsilon t \rightarrow 0) \simeq \omega \simeq 1+\varepsilon t / 2,
$$

where the last follows from the basic assumption generating this class of solution. These forms (2.28) would have to coincide with approximations to the inner solutions found in Section 3 below for $t$ in a range $\varepsilon^{-1 / 2} \ll t \ll$ $\varepsilon^{-1}$ in order that sustained resonant solutions be available from accessible perturbation conditions at $-\infty$, but it will be seen in Section 3 that this matching condition cannot be met. In fact the approximation (2.25) to the sustained resonant solution can be continued to the left of $\omega=1$ (that is, to the left of $t=0$ ) before it breaks down, and clearly it cannot generate a $p$ (through 2.27) compatible with an outer solution of the type (2.16).

By contrast with the previous case, the limit forms of the approximations $(2.15-17)$ are not fixed by the relative phase of the $x$ oscillation and $\sin (\psi)$ but are

$$
\begin{aligned}
& x\left(\varepsilon t \rightarrow 0_{ \pm}\right) \simeq 2 \operatorname{Re}\left\{A_{ \pm}\left(\frac{p_{ \pm}^{2}+1}{2}\right)^{-1 / 4} \exp \left( \pm i\left(1+\left(p_{ \pm}-1\right) / 2\right) t\right)\right\} \\
& \psi\left(\varepsilon t \rightarrow 0_{ \pm}\right) \simeq p_{ \pm} t+\psi_{1 \pm} \\
& p\left(\varepsilon t \rightarrow 0_{ \pm}\right) \simeq p_{ \pm}
\end{aligned}
$$

once again applying on intervals $\varepsilon^{-1 / 2} \ll t \ll \varepsilon^{-1}$. Here it is remarked that the apparently inconsequential difference between the third member of 
(2.28) and (2.31) is in fact crucial in deciding the possibility or otherwise of matching inner and outer solutions.

\section{The inner solution-transient resonance}

It is perhaps helpful at this stage to give a qualitative account of the processes involved at the transient resonance (that is, in an $O\left(\varepsilon^{-1 / 2}\right)$ interval of $t$ about $t=0$ ) from an energetics standpoint. If $\psi$ replaces $t$ as the independent variable in (2.2-5) then that system becomes

$$
\begin{gathered}
\frac{d^{2} x}{d \psi^{2}}+\varepsilon x \sin (\psi) \frac{\omega^{2}}{p^{2}} \frac{d x}{d \psi}+\frac{1}{2}\left(1+\frac{\omega^{2}}{p^{2}}\right) x=0, \\
\frac{d p^{2}}{d \psi}=2 \varepsilon x \sin (\psi) \omega^{2}
\end{gathered}
$$

where $t$ is calculated from the integral

$$
t=\int_{0}^{\psi} d \psi / p(\psi)
$$

The dependence of $\omega$ upon $\psi$ is not explicit, but this is not important since $p$ is slowly varying (3.2) and so $\psi$ and $t$ are roughly linearly connected. Further, on intervals for which $p$ is close to $\omega$, that is, in particular near $t=0,(3.1)$ can be regarded as describing a weakly non-linear harmonic oscillator

$$
\frac{d^{2} x}{d \psi^{2}}+x=-\varepsilon x \sin (\psi) \frac{d x}{d \psi}
$$

whose solutions are of the form $x=\alpha \sin (\psi+\Psi)$, where $\alpha$ and $\Psi$ are slowly varying functions of $\psi$. Then the usual energetic form

$$
\frac{d}{d \psi}\left(\left(\frac{d x}{d \psi}\right)^{2}+x^{2}\right)=-2 \varepsilon x \sin (\psi)\left(\frac{d x}{d \psi}\right)^{2}
$$

would interpret the right hand side of (3.4) as indicating a mean energy flow rate, depending on the average of $(x \sin \psi)$ on the fast time scale

$$
\text { average }(-x \sin (\psi))=-\alpha \cos (\Psi) / 2 \text {. }
$$

Similarly, the right hand side of (3.2) indicates an energy flow rate for the spin mode, in the opposite sense to that for the pitch/yaw. (It is something of a paradox that the solution which locally corresponds to the sustained resonance case discussed above in Section 2, $x=-|\alpha| \cos (\psi+\psi(0))$, has zero average energy exchange rate at the transient resonance.) These observations agree with intuition: in the zero applied moment case for the axi-symmetric projectile, if some mechanism caused configuration change at constant energy 
and momentum, increase in spin energy would imply a decrease in energy associated with pitch/yaw (precession), and vice-versa.

The next step is to put the ideas outlined above into a rigorous mathematical argument. It is convenient to first introduce a new variable $\Pi$ through the identification

$$
p=1+\varepsilon \Pi, \quad \Pi(0)=0 .
$$

The implied scaling is not, in fact, the correct one- $\Pi$ turns out ultimately to be $O\left(\varepsilon^{-1 / 2}\right)$-but it gives a tidier presentation to proceed as indicated.

With the object of finding approximate solutions whose $t$ interval of applicability extends to $|t| \gg \varepsilon^{-1 / 2}$, the independent variable transformation $t \rightarrow \varphi$ is introduced (in a parallel to the Lighthill [7] technique) through the locally near identity transformation

$$
\frac{d \varphi}{d t}=p(1+\varepsilon L)=(1+\varepsilon \Pi)(1+\varepsilon L),
$$

where $L$ is chosen so that formally

$$
\left(\frac{d \varphi}{d t}\right)^{-2}\left(p^{2}+\omega^{2}(\varepsilon t)\right)=1+\omega^{2}(\varepsilon \varphi)+O\left(\varepsilon^{3}\right)
$$

Then $L$ is a formal power series

$$
\varepsilon L(t)=\sum_{1}^{\infty} \varepsilon^{j} L_{j}(t)
$$

with

$$
\begin{aligned}
& L_{1}=-\omega^{2} \Pi /\left(1+\omega^{2}\right) \\
& L_{2}=\left(3 \Pi^{2} \omega^{2}-2 \omega^{2} \int_{0}^{t}\left(L_{1}+\Pi\right) d t-L_{1}^{2}\left(1+\omega^{2}\right)\right) /\left(2\left(1+\omega^{2}\right)\right)
\end{aligned}
$$

etc., and so $\varphi$ can be expressed as

$$
\varphi(t)=t+\varepsilon \int_{0}^{t} \Pi\left(1+\omega^{2}\right)^{-1} d t+O\left(\varepsilon^{2}\right) .
$$

The transformation (3.6-7) is locally invertible, and the inverse is

$$
t(\varphi)=\varphi-\varepsilon \int_{0}^{\varphi} \Pi\left(1+\omega^{2}\right)^{-1} d \varphi+O\left(\varepsilon^{2}\right)
$$

while the $\psi(\varphi)$ relation is

$$
\psi=\varphi+\psi_{0}+\varepsilon \int_{0}^{\varphi} \omega^{2} \Pi\left(1+\omega^{2}\right)^{-1} d \varphi+O\left(\varepsilon^{2}\right),
$$

if the initial value is denoted as $\psi(0)=\psi_{0}$. All of the approximations (3.1113) are locally near linear. The error orders given in them are formal, and 
actually underestimate as a consequence of the underestimation of the magnitude of $\Pi$. The effect on the analysis will be reviewed later.

On implementation of the transformation (3.6-7), (2.2-3) become

$$
\begin{gathered}
\frac{d^{2} x}{d \varphi^{2}}+\frac{\varepsilon x \omega^{2} \sin \left(\varphi+\psi_{0}\right)}{1+\omega^{2}} \frac{d x}{d \varphi}+\frac{1}{2}\left(1+\omega^{2}\right) x=\left\{\varepsilon^{2} x \frac{d x}{d \varphi} \cdots\right\}+\left\{\varepsilon^{3} x \cdots\right\} \\
\varepsilon \frac{d \Pi}{d p}=\varepsilon \omega^{2} x \sin \left(\varphi+\psi_{0}\right)+\left\{\varepsilon^{2} \cdots\right\}
\end{gathered}
$$

where $\omega^{2}(\varepsilon \varphi)=\exp (\varepsilon \varphi)$ and where the \{\} braces denote the terms omitted in the subsequent approximating analysis. Again, the magnitudes indicated by the formal powers of $\varepsilon$ are underestimates, but the true magnitudes are small enough to justify the omissions.

The reduced system $(3.14-15)$ is therefore

$$
\begin{gathered}
\frac{d^{2} x}{d \varphi^{2}}+\frac{\varepsilon x \omega^{2} \sin \left(\varphi+\psi_{0}\right)}{1+\omega^{2}} \frac{d x}{d \varphi}+\frac{1}{2}\left(1+\omega^{2}\right) x=0, \\
\varepsilon \frac{d \Pi}{d \varphi}=\varepsilon \omega^{2} x \sin \left(\varphi+\psi_{0}\right)
\end{gathered}
$$

and its essential feature is the absence of the variable $\Pi$ from its first member. Not only does this reduce the problem to second order plus two quadratures, but also it will be seen to give approximation validity on the interval required for matching to the outer solutions of Section 2 , that is, $|\varphi|$ intervals $\sim O\left(\varepsilon^{-1 / 2-q}\right)$ for some positive $q$ (but $q<1 / 2$ ).

In oscillatory problems in dynamics it is usually an advantage to work in complex notation, the advantage being that otherwise necessarily additive error statements can be expressed multiplicatively, and this is generally desirable. The practice is follows below, and it leads naturally to a particular formalism.

Solutions $X$ and $\bar{X}$ (where the overbar is used to denote complex conjugation) of the linear equation

$$
\frac{d^{2} X}{d \varphi^{2}}+\frac{1}{2}(1+\exp (\varepsilon \varphi)) X=0
$$

which dominates (3.16), may be considered to be known. Their expansions, asymptotic to an arbitrary power of $\varepsilon$, may be constructed using the Liouville-Green methods described by Olver [8]. The rapid growth of the exponential in the coefficient at $+\infty$, and its decay at $-\infty$, allows strong uniform error statements to be made. It is convenient to express the solutions in terms of a function

$$
\theta(\varepsilon \varphi)=\int_{0}^{\varepsilon \varphi} \theta^{\prime}(u) d u
$$


whose derivative is

$$
\theta^{\prime}(u)=2^{-1 / 2}(1+\exp (u))^{1 / 2}
$$

The solutions of (3.18) can then be inferred from the expressions

$$
\begin{aligned}
X & =\left(\theta^{\prime}(\varepsilon \varphi)\right)^{-1 / 2}(1+\varepsilon E) \exp (i \theta(\varepsilon \varphi) / \varepsilon), \\
\frac{d X}{d \varphi} & =\left(\theta^{\prime}(\varepsilon \varphi)\right)^{1 / 2} i(1+\varepsilon F) \exp (i \theta(\varepsilon \varphi) / \varepsilon),
\end{aligned}
$$

where the errors $E$ and $F$ satisfy bounds

$$
|\varepsilon E|,|\varepsilon F| \leq \exp (2 \varepsilon \Lambda)-1
$$

with the exponent

$$
\Lambda(\varepsilon \varphi)=\int_{0}^{\varepsilon \varphi}\left(\theta^{\prime}(u)\right)^{-1 / 2} \frac{d^{2}}{d u^{2}}\left(\left(\theta^{\prime}(u)\right)^{-1 / 2}\right) d u
$$

is uniformly bounded for all values of the argument. So the bounds (3.23) can be further simplified as

$$
|\varepsilon E|,|\varepsilon F| \sim O(\varepsilon) .
$$

Express solutions of (3.16) as

$$
x=y X+\bar{y} \bar{X}
$$

where the real and imaginary parts of $y$ are to be determined. An extra condition must thus be imposed to close the problem, and it is taken to be

$$
\frac{d y}{d \varphi} X+\frac{d \bar{y}}{d \varphi} \bar{X}=0
$$

With this condition the derivative is

$$
\frac{d x}{d \varphi}=y \frac{d X}{d \varphi}+\bar{y} \frac{d \bar{X}}{d \varphi}=i \theta^{\prime}(y X(1+O(\varepsilon))-\bar{y} \bar{X}(1+O(\varepsilon))),
$$

the reduction coming from the properties $(3.21-25)$ of $X$.

After substituting (3.21) and (3.28) for $X$ and its derivative in (3.16), and on calling the condition (3.27), it follows that

$$
\begin{aligned}
\frac{d^{2} x}{d \varphi^{2}} & +\frac{1}{2}\left(1+\omega^{2}\right) x=2 i \frac{d y}{d \varphi} \theta^{\prime} X(1+O(\varepsilon)) \\
& =-\frac{i \varepsilon \theta^{\prime}(y X+\bar{y} \bar{X})(y X(1+O(\varepsilon))-\bar{y} \bar{X}(1+O(\varepsilon))) \omega^{2} \sin \left(\varphi+\psi_{0}\right)}{1+\omega^{2}}
\end{aligned}
$$


(together with its conjugate, if required) can be used to determine $y$. Further, since $X$ has no zeros for real $\varphi$ it can be divided out, and if only the largerorder terms are retained in the resulting expression, it is

$$
\begin{gathered}
\frac{d y}{d \varphi}=-\frac{\varepsilon}{2} \omega^{2}\left(y^{2} X \sin \left(\varphi+\psi_{0}\right)-\bar{y}^{2} \bar{X}^{2} X^{-1} \sin \left(\varphi+\psi_{0}\right)\right)\left(1+\omega^{2}\right)^{-1} \\
=-\frac{\varepsilon}{2} \omega^{2}\left(-y^{2} X \exp \left(-i\left(\varphi+\psi_{0}\right)\right)-2 i \bar{y}^{2} \bar{X}^{2} X^{-1} \sin \left(\varphi+\psi_{0}\right)\right. \\
\left.\quad+y^{2} X \exp \left(i\left(\varphi+\psi_{0}\right)\right)\right)\left(2 i\left(1+\omega^{2}\right)\right)^{-1}
\end{gathered}
$$

On substituting the evaluation (3.21) for $X$ in (3.30), it will be seen that the terms on the right-hand side of the latter are of two essentially different kinds. Ignoring for the moment the slowly-varying factors which are powers of $\theta^{\prime}$ and $\omega$, the second pair in the large brackets have explicit, fast $\varphi$ dependences that can be expressed as the derivatives of asymptotic series with no large multiplying factors. For example, the first such term can be split into a pair, one of which, again for example, has the fast dependence

$$
\begin{aligned}
\bar{X}^{2} X^{-1} & \exp \left(i\left(\varphi+\psi_{0}\right)\right) \\
& =\text { (slowly varying factors) }\left(\exp \left(i\left(\psi_{0}-\frac{3 \theta-\varepsilon \varphi}{\varepsilon}\right)\right)\right),
\end{aligned}
$$

where $\theta$ is defined in (3.20). Now, on any interval on which the derivative $P^{\prime}$ of some given $P$ does not vanish, any product of terms

$$
Q(\varepsilon \varphi) \exp (i P(\varepsilon \varphi) / \varepsilon)
$$

can be expressed as the derivative of an asymptotic series

$$
\begin{aligned}
& Q(\varepsilon \varphi) \exp (i P(\varepsilon \varphi) / \varepsilon) \\
& =\frac{d}{d \varphi}\left(\left\{-i\left(\frac{Q}{P^{\prime}}\right)+\frac{\varepsilon}{P^{\prime}}\left(\frac{Q}{P^{\prime}}\right)^{\prime}-i \frac{\varepsilon}{P^{\prime}}\left(\frac{\varepsilon}{P^{\prime}}\left(\frac{Q}{P^{\prime}}\right)^{\prime}\right)^{\prime} \ldots\right\} \exp (i P / \varepsilon)\right)
\end{aligned}
$$

and any term in (3.30) containing such a factor satisfies the conditions described in the next section which enables it to be, so to say, averaged out. The last two terms in the large brackets in (3.30) are expressible as the sum of such terms, and thus can be ignored in the primary averaging procedure.

Thus the averaged version of (3.30) is

$$
\frac{d y}{d \varphi}=-\frac{i \varepsilon}{4} \omega^{2}\left(1+\omega^{2}\right)^{-1} y^{2} X \exp \left(-i\left(\varphi+\psi_{0}\right)\right)
$$

or, to leading order

$$
\frac{d y^{-1}}{d \varphi}=\frac{i \varepsilon}{4} \omega^{2}\left(1+\omega^{2}\right)^{-1}\left(\theta^{\prime}\right)^{-1 / 2} \exp \left(i\left(\frac{\theta(\varepsilon \varphi)-\varepsilon \varphi}{\varepsilon}-\psi_{0}\right)\right) .
$$


This last equation is closely related to the first of Kath's [3] equations (2.14), but the essential difference is that the preliminary transformation (3.6-7) here has made the oscillatory factor in (3.33) known in terms of the $\varphi$ variable. Of course, the solution of the problem has to be completed before $\varphi$ itself can be expressed in terms of the original $t$ variable.

Standard asymptotic-approximation methods can be used to evaluate integrals of the type

$$
I(\varphi)=\int_{0}^{\varphi} Q(\varepsilon \varphi) \exp (i R(\varepsilon \varphi) / \varepsilon) d \varphi
$$

when $R$ and $Q$ are real on the real line and analytic in a non-small origincentered disk in the complex $(\varepsilon \varphi)$ plane, in cases when $R(0)=R^{\prime}(0)=0$. These evaluations are expressed in terms of complex Fresnel integrals [9]

$$
\begin{aligned}
\int_{0}^{\mu} \exp \left(i \varepsilon c \mu^{2}\right) d \mu & =(\varepsilon c)^{-1 / 2} \int_{0}^{\mu \sqrt{c \varepsilon}} \exp \left(i z^{2}\right) d z \quad(c>0) \\
& \equiv(c \varepsilon)^{-1 / 2} F(\mu \sqrt{c \varepsilon}),
\end{aligned}
$$

and it can be shown (see, for example [1]) that they are

$$
I(\varepsilon \varphi)=Q(0)\left(R^{\prime \prime}(0) \varepsilon / 2\right)^{-1 / 2}\left(F\left(\left(R^{\prime \prime}(0) \varepsilon / 2\right)^{1 / 2} \varphi\right)+O\left(|\varphi|^{2} \varepsilon^{2}\right)\right) .
$$

The error term in (3.36) finds the approximation to be a good one on any interval $|\varphi| \sim O\left(\varepsilon^{-1+q}\right)$ for any $q>0$; that is, an interval large enough that $F$ can approach its asymptotic value

$$
F( \pm \infty)= \pm \sqrt{\pi / 8} \exp (i \pi / 4)
$$

For the solution of (3.33) straightforward calculations give

$$
Q(0)=\frac{i \varepsilon}{8} \exp \left(-i \psi_{0}\right), \quad R^{\prime \prime}(0) / 2=1 / 8
$$

in (3.36), so the result is

$$
\begin{aligned}
y & \simeq\left([y(0)]^{-1}+i(\varepsilon / 8)^{1 / 2} \exp \left(-i \psi_{0}\right) F\left((\varepsilon / 8)^{1 / 2} \varphi\right)\right)^{-1} \\
& \simeq y(0)-[y(0)]^{2} i(\varepsilon / 8)^{1 / 2} \exp \left(-i \psi_{0}\right) F\left((\varepsilon / 8)^{1 / 2} \varphi\right) .
\end{aligned}
$$

The error statement is omitted from these results; it can be taken from the asymptotic approximation (3.36). While the first line of (3.39) is the more attractive, error control on the required $|\varphi|$ interval demands that $\varepsilon$ is small, so the second line of the result is really the only admissible one. (It will be shown below, on calculating $p$ and $\Pi$, that the solution singularity suggested by the first line of (3.39) could not in any case be reached in the $t$ variable.)

On substituting (3.26) for $x$ in (3.17), arguments analogous to those used to reduce (3.30) reveal that an approximation to $\Pi$ is obtained from the 
integration

$$
\varepsilon \Pi \simeq-\varepsilon \operatorname{Im}\left\{\int_{0}^{\varphi} y \omega^{2} X \exp \left(-i\left(\varphi+\psi_{0}\right)\right) d \varphi\right\} .
$$

Equation (3.32) shows that

$$
\varepsilon \omega^{2} X \exp \left(-i\left(\varphi+\psi_{0}\right)\right)=4 i\left(1+\omega^{2}\right) y^{-2} \frac{d y}{d \varphi}
$$

and in the $o\left(\varepsilon^{-1}\right)$ neighbourhood of the origin where this calculation is done repeated partial integration gives the result

$$
\begin{aligned}
\varepsilon \Pi & =8 \operatorname{Re}\{\ln (y(0) / y(\varphi))+O(\varepsilon)\} \\
& \simeq 8 \operatorname{Re}\left\{\ln \left(1+y(0) i(\varepsilon / 8)^{1 / 2} \exp \left(-i \psi_{0}\right) F\left((\varepsilon / 8)^{1 / 2} \varphi\right)\right)\right\} \\
& \simeq 8 \operatorname{Re}\left\{y(0) i(\varepsilon / 8)^{1 / 2} \exp \left(-i \psi_{0}\right) F\left((\varepsilon / 8)^{1 / 2} \varphi\right)\right\},
\end{aligned}
$$

in which has been used the evaluation (3.39) of $y$. It is stressed that the error term in the first line of this result is not linear in $t$, being in fact $\left\{\varepsilon \times\right.$ (uniformly bounded function) $\left.+O\left(\varepsilon^{2}\right)\right\}$; and that the last line is the usable result-for the same reasons advanced in the discussion of the result (3.39).

It is also noted that, questions of solution admissibility for the moment ignored, the second line of (3.41) implies that a zero of $p=1+\varepsilon \Pi$ must immediately precede the singularity in the logarithm at the zero of its argument. The underlying variable transformation (3.6) would be singular at the value of $\varphi=\Phi$ corresponding to the zero of $p$, so that $t(\Phi)$ is the point at infinity. Thus the singularities in $y$ and $p$ suggested by the first lines of their evaluations $(3.39,3.41)$ are not reachable to finite $t$.

The problem is now that of matching the "large $\left|\varepsilon^{1 / 2} \varphi\right|$ " forms of the evaluations $(3.39,3.41)$ to the limit forms $(2.29-31)$ of the outer solutions (2.15-17). The start point for the calculation is finding explicit approximations to the basic transformation (3.6), and in particular the integral in the expression (3.12). For suitably large values of $\left|\varepsilon^{1 / 2} \varphi\right|$ 一that is, much greater than unity but much less than $\varepsilon^{-1 / 2}$-this can be approximated as

$$
\begin{aligned}
\varepsilon \int_{0}^{\varphi} \Pi\left(1+\omega^{2}\right)^{-1} d \varphi & \simeq \varepsilon \int_{0}^{\varphi} \Pi d \varphi / 2 \\
& \simeq 4 \operatorname{Re}\left\{i y(0) \exp \left(-i \psi_{0}\right) \int_{0}^{(\varepsilon / 8)^{1 / 2} \varphi} F(w) d w\right\} \\
& \simeq 4 \operatorname{Re}\left\{i y(0) \exp \left(-i \psi_{0}\right)\left((\varepsilon / 8)^{1 / 2} \varphi F(\infty \operatorname{sgn} \varphi)-i / 2\right)\right\} \\
& \equiv \frac{\left(\mu_{ \pm}-1\right)}{2} \varphi+\sigma,
\end{aligned}
$$


where the notation

$$
\lim _{\varphi \rightarrow \pm \infty} \Pi(\varphi)=\mu_{ \pm}-1 \sim O\left(\varepsilon^{1 / 2}\right)
$$

is introduced, and

$$
\sigma\left(y(0), \psi_{0}\right)=2 \operatorname{Re}\left\{y(0) \exp \left(-i \psi_{0}\right)\right\}
$$

is defined. Then the transformation (3.12) can be asymptotically expressed as

$$
t \simeq \varphi\left(1-\frac{\mu_{ \pm}-1}{2}\right)-\sigma,
$$

$\left(\varepsilon^{1 / 2} t, \varepsilon^{1 / 2} \varphi \rightarrow \pm \infty\right)$ and the inverse is asymptotically

$$
\varphi \simeq t\left(1+\frac{\mu_{ \pm}-1}{2}\right)+\sigma
$$

in the same limits. Finally, the integral in the approximation (3.13) can be asymptotically approximated to yield in these limits

$$
\begin{aligned}
\psi & \simeq \varphi\left(1+\frac{\mu_{ \pm}-1}{2}\right)+\psi_{0}+\sigma \\
& \simeq t \rho_{ \pm}+\psi_{0}+2 \sigma .
\end{aligned}
$$

The last set of approximations enables the matching procedure to be carried out after calculating the limit forms of the results of this section:

$$
\begin{gathered}
x\left(\varepsilon^{1 / 2} \varphi \rightarrow \pm \infty\right)=2 \operatorname{Re}\{y X\} \simeq 2 \operatorname{Re}\left\{y_{ \pm} \exp (i \varphi)\right\} \\
\simeq 2 \operatorname{Re}\left\{y_{ \pm} \exp \left(i\left(1+\frac{\mu_{ \pm}-1}{2}\right) t+i \sigma\right)\right\}, \\
p\left(\varepsilon^{1 / 2} \varphi \rightarrow \pm \infty\right) \simeq \rho_{ \pm} .
\end{gathered}
$$

In these last results, $y_{ \pm}$denotes the indicated limit, explicitly

$$
\begin{gathered}
y_{ \pm}=y(0)\left(1 \mp 8^{-1} y(0)(\pi \varepsilon)^{1 / 2} \exp \left(-i\left(\psi_{0}-\pi / 4\right)\right)\right), \\
\mu_{ \pm}=1 \pm(\pi \varepsilon)^{1 / 2} \operatorname{Re}\left\{y(0) \exp \left(-i\left(\psi_{0}-3 \pi / 4\right)\right)\right\},
\end{gathered}
$$

following from the approximations $(3.37,3.39,3.41)$. (For sufficiently small $\varepsilon,(3.49)$ has a root $y(0)$ close to $y_{-}$, in particular for all $\psi_{0} \in[0,2 \pi)$, so a $y(0)$ can be calculated for bounded but otherwise arbitrary $y_{-}$.)

The matching of the outer approximations (2.15-17) whose limit forms are (2.29-31) to the inner approximations implied by (3.39) and (3.41) through the limit forms $(3.47,3.46$ and 3.48$)$ follows if the constants $A_{+}, A_{-}, p_{+}$, $p_{-}, \psi_{1+}$, and $\psi_{1-}$ are chosen so that

$$
p_{+}=\mu_{+} ; \quad p_{-}=\mu_{-},
$$




$$
\begin{gathered}
A_{+}\left(\frac{p_{+}^{2}+1}{2}\right)^{-1 / 4}=y_{+} \exp (i \sigma) ; \quad A_{-}\left(\frac{p_{-}^{2}+1}{2}\right)^{-1 / 4}=y_{-} \exp (i \sigma) \\
\psi_{1+}=\psi_{1-}=\psi_{0}+2 \sigma
\end{gathered}
$$

On the other hand, the presence of the term $(\varepsilon t / 2)$ in the third of the limit forms (2.28) precludes the matching of the corresponding approximate solutions with those of the limit forms $(3.46-48)$. It is therefore to be concluded that the inner solutions calculated here do not show the phenomenon of sustained resonance-at least, for sufficiently small $\varepsilon$.

To "complete" the problem, it remains to show that there is a continuous, one-to-one, onto relationship between a set of parameters describing all possible dynamic states at the origin, and a set describing all possible initial dynamical states. The matching conditions (3.51-53) show that this reduces to showing a continuous, one-to-one, onto relationship between the origin set, and a set describing the asymptotic dynamical state at the negative limit for the inner solution, which is now referred to as the "initial" state. An approximate description of the relationship is now given; once the approximation is settled, it can be corrected in a straightforward way-at least, in principle.

The fourth-order system's (2.2-4) dynamical configuration at the origin (the "original configuration") is determined by three free real parameters namely $\psi_{0}$, which locates the projectile's mass center eccentricity with respect to some datum, and $\vartheta$ and $\xi$ where

$$
\mathcal{y}(0) \equiv \vartheta \exp (i \xi) \text {. }
$$

To this list must be added the fixed original condition (2.6) on $p(0)$.

The initial configuration is determined by real parameters $\psi_{1-}, \lambda, \alpha$ and $\beta$, where $\lambda$ is a measure of the spin perturbation

$$
\lambda=\varepsilon^{-1 / 2}\left(1-\mu_{-}\right)
$$

and $\alpha$ and $\beta$ are the amplitude and phase in the matching requirement (3.52) which is approximately

$$
\alpha \exp (i \beta)=y_{-} \exp (i \sigma) .
$$

Not all the nominated constants can be independent, because $p(0)$ is specified in the original configuration, and so there must be one relationship among the initial configuration variables.

Now (3.49) shows that

$$
y(0) \approx y_{-}
$$

and therefore

$$
\alpha \approx \vartheta
$$


and to sufficient an approximation, (3.56) then reduces to

$$
\beta \approx \xi+\sigma=\xi+2 \vartheta \cos \left(\psi_{0}-\xi\right)
$$

while $(3.50,3.55)$ are together

$$
\lambda=\pi^{1 / 2} \vartheta \cos \left(\psi_{0}-\xi-3 \pi / 4\right) .
$$

Thus for any triple $\left(\psi_{0}, \theta, \xi\right)$ there can be calculated a triple $(\lambda, \alpha, \beta)$. But closer examination of $(3.53,3.59-60)$ suggests that the relative phases

$$
\hat{\beta}=\beta-\psi_{0}, \quad \hat{\xi}=\xi-\psi_{0}
$$

along with $\alpha$ and $\lambda$, are the effective variables at the initial state. This is so because, on using (3.53) and the definitions (3.61), (3.59-60) become a parametric relationship connecting $\hat{\beta}$ and $\lambda$

$$
\begin{gathered}
\hat{\beta}=\hat{\xi}+2 \vartheta \cos (\hat{\xi}) \equiv \hat{\beta}(\hat{\xi}), \\
\lambda=\pi^{1 / 2} \vartheta \cos (\hat{\xi}+3 \pi / 4),
\end{gathered}
$$

and it can be shown that, given any fixed non-negative $\vartheta$, the equations

$$
\hat{\beta}\left(\hat{\xi}_{1}\right)=\hat{\beta}\left(\hat{\xi}_{2}\right) \text { and } \lambda\left(\hat{\xi}_{1}\right)=\lambda\left(\hat{\xi}_{2}\right)
$$

have a unique solution $\hat{\xi}_{1}=\hat{\xi}_{2}$ on a $2 \pi$ interval of $\hat{\xi}$

$$
-7 \pi / 4 \leq \hat{\xi}<\pi / 4
$$

that suffices to describe all possible original relative phases. For the original state problem then, in the light of $(3.58,3.62-63)$ it is only necessary to prescribe the three original parameters $\psi_{0}, \vartheta$ and $\hat{\xi}$ and then all the initial parameters can be inferred uniquely. The other, now subsidiary, original parameter $\xi$ is obtainable from (3.61).

Thus it is better to interpret the original-value problem in the following geometric way. To keep the imagery at its most simple, assume for the moment that $\vartheta$ and hence $\alpha$, approximately, are fixed. Then the solution to the original-value problem can be regarded as a one-to-one continuous map from points

$$
0 \leq \psi_{0}<2 \pi, \quad-7 \pi / 4 \leq \hat{\xi}<\pi / 4
$$

in the plane in $\mathbb{R}^{3}\left(\xi, \hat{\xi}, \psi_{0}\right)$

$$
\xi-\psi_{0}-\hat{\xi}=0
$$

onto a set of points on the surface of a cylinder in $\mathbb{R}^{3}(\beta, \hat{\beta}, \lambda)$ defined parametrically (parameter $\tau$ )

$$
\begin{gathered}
\hat{\beta}=\tau+2 \vartheta \cos (\tau), \\
\lambda=\pi^{1 / 2} \vartheta \cos (\tau+3 \pi / 4), \\
-7 \pi / 4 \leq \tau<\pi / 4 ;
\end{gathered}
$$


the mapping is described by (3.62-63), and by (3.61) in the form

$$
\beta=\hat{\beta}(\hat{\xi})+\psi_{0}
$$

The point set accessed from the original state is the set $\mathscr{S}_{\theta}$ cut off from the surface of the cylinder (3.67) by the planes

$$
\beta=\hat{\beta} \quad \text { and } \quad \beta=\hat{\beta}+2 \pi .
$$

All possible states at the origin are thus uniquely and continuously associated with points on $\mathscr{S}_{\theta}$, and conversely there are available at least $2 \pi$ intervals of $\hat{\xi}$ and $\hat{\beta}$.

For the initial-state problem, $\alpha>0$ can be prescribed arbitrarily. Then, by virtue of the approximation (3.58), the set $\mathscr{S}_{\theta}$ is known with the value of $\vartheta$ taken from that approximation to be $\vartheta=\alpha$, and a complete set of states at the origin can be reached, each uniquely associated with a point $(\beta, \hat{\beta}, \lambda)$ of $\mathscr{S}_{\alpha}$. For, given some point $(\beta, \hat{\beta}, \lambda)$ on $\mathscr{S}_{\alpha}$, and noting that a $2 \pi$ range of values of $\beta$ is available with any $(\hat{\beta}, \lambda)$ pair, the original state $\left(\xi, \hat{\xi}, \psi_{0}\right)$ is found using the unique solution $\tau=\hat{\xi}$ of the equations

$$
\begin{aligned}
\hat{\beta}= & \tau+2 \vartheta \cos (\tau), \\
\lambda= & \pi^{1 / 2} \vartheta \cos (\tau+3 \pi / 4), \\
& -7 \pi / 4 \leq \tau<\pi / 4 .
\end{aligned}
$$

(Both equations (3.71) are needed to specify a unique $\hat{\xi}$, as either on its own will not suffice.) Then the remaining initial configuration values are

$$
\psi_{0}=\beta-\hat{\beta} \text { and } \xi=\hat{\xi}+\psi_{0} .
$$

The foregoing is an approximate description of the geometric connection between three degree-of-freedom initial- and final-value problems, which in its most concise mathematical form describes a continuous one-to-one mapping between a set of parameters $\left(\vartheta, \hat{\xi}, \psi_{0}\right)$ sufficient to describe all original states, and a set $(\alpha, \tau, \beta)$ sufficient to describe all initial states. The mapping is approximated by the equations

$$
\alpha=\vartheta, \quad \tau=\hat{\xi}, \quad \beta=\hat{\beta}(\hat{\xi})-\psi_{0}
$$

on $0 \leq \vartheta,-7 \pi / 4 \leq \hat{\xi}<\pi / 4,0 \leq \psi_{0}<2 \pi$; and the inverse map inverts the third member of (3.74)

$$
\psi_{0}=\hat{\beta}(\tau)-\beta
$$

on $0 \leq \alpha,-7 \pi / 4 \leq \tau<\pi / 4,(\hat{\beta}(\tau)-2 \pi)<\beta \leq \hat{\beta}(\tau)$. 
They (3.74-75) are correctable for the errors induced by the approximations (notably 3.56-58) made in their construction. All possible perturbation states and configurations are describable and all possible perturbation states and configurations are continuously reachable for either the original or initial problems, so the description is complete. (Of course, the problem before the introduction of the scaling (2.1) is fourth order; and the missing degree of freedom is the scale factor $K$ introduced in the variable scalings (2.1).) It is therefore concluded that the sustained resonant solutions discussed in Section 2 are not associated with perturbation initial conditions.

Finally, it is remarked that the origin shifting used in conjunction with the scaling (2.1) to reduce the Kevorkian model to a less awkward form has a very distorting effect on the above results if any attempt is made to restore it to the analysis. The difficulty is that, for example, an $O\left(\varepsilon^{-1 / 2}\right)$ shift in the origin leads only to an $O\left(\varepsilon^{1 / 2}\right)$ change in the analysis.

\section{Existence}

In this section, an existence theory for a family of transiently resonant oscillators is given. The theory is modelled on that for averaged systems, a succinct account of which is contained in the text by Guckenheimer and Holmes [2], and the preamble together with part (1) of their Theorem 4.1.1 is relevant.

Suppose an initial-value problem for a complex-valued variable $y$ of a real variable $t$ is

$$
\begin{gathered}
\frac{d y}{d t}=\delta f(y) C\left(\varepsilon^{1 / 2} t\right)+\delta g(y, \bar{y}, t), \quad 0<|\delta|, \varepsilon \ll 1, \\
y(0)=\alpha .
\end{gathered}
$$

The non-autonomous multiplier $C$ could in practice have a more complicated $t$-dependence (as in the example of Section 3 above, where $C$ also has an $\varepsilon t$ variation) but the arguments which will be given are adequate provided the secondary variation is on a substantially slower scale, and $C$ is subject to some other restrictions which are described below.

In the application considered here, $f$ and $g$ are entire functions of their separate arguments for all $t$. Then a $w(y, \bar{y}, t)$ can be constructed with the property

$$
\frac{\partial w}{\partial t}=g, \quad w(t=0)=0 .
$$

It is assumed that the following conditions are satisfied: there exist real constants $\mathscr{K}$ and $Z$ such that for all $t$ and points $y$ in a disk $\mathscr{D}_{1}$ in the complex plane 


$$
\left\{y \in \mathscr{D}_{1}:|y| \leq Z+\delta \mathscr{K}\right\} \text {, }
$$

the bounds

$$
\max \left\{|w|,\left|2 \frac{\partial g}{\partial y}\right|,\left|2 \frac{\partial g}{\partial \bar{y}}\right|,\left|\frac{d f}{d y}\right|,|C|\right\} \leq \mathscr{K}
$$

apply. The complex-valued $C(u)$ is further supposed to be integrable for all $u$, its integral having finite limits as $u \rightarrow \pm \infty$. It would be a not unusual case if $C$ were $C(u)=\exp \left(i u^{2}\right)$.

Suppose $\delta$ is specified as

$$
\delta=\varepsilon^{(Q+1 / 2)}
$$

for $Q$ positive. Then, for any initial value $\alpha \in \mathscr{D}_{1}$ of $y$ and sufficiently small $\varepsilon$, on any interval

$$
|t \delta| \sim o(1),
$$

solutions of the differential equation (4.1) are uniformly approximated by $z_{0}(t)$ which satisfies

$$
\frac{d z_{0}}{d t}=\delta f\left(z_{0}\right) C\left(\varepsilon^{1 / 2} t\right)
$$

Thus, if $H$ is defined as a natural indefinite integral (here a "natural" indefinite integral is one such as

$$
\left.\int u^{n} d u=u^{n+1} /(n+1)\right)
$$

then

$$
\int_{\alpha}^{z_{0}} d u / f(u)=H\left(z_{0}\right)-H(\alpha)
$$

and if also $\Gamma$ is defined to be

$$
\varepsilon^{-1 / 2} \delta \int_{0}^{\varepsilon^{1 / 2} t} C(u) d u \equiv \varepsilon^{Q} \Gamma\left(\varepsilon^{1 / 2} t\right)
$$

$z_{0}$ can be expressed in terms of an inverse function $\mathrm{H}^{-1}$

$$
z_{0}(t)=H^{-1}\left(H(\alpha)+\varepsilon^{Q} \Gamma\left(\varepsilon^{1 / 2} t\right)\right) .
$$

Remarks. 1. The first term in (4.1) is analogous to the averaged term in the usual theory, and the second one to the residual which, in that theory, has zero $t$-average.

2. The construction of the approximate solution implies the existence of a bound for

$$
\left|f\left(z_{0}(t)\right)\right|^{-1} \leq \mathscr{K}
$$

$\mathscr{K}$ being redefined if necessary. 
3. Error statements justifying the above claims will be made later.

Following the averaging procedure, introduce a new dependent variable $z$ by means of the family of near identity transformations

$$
\begin{aligned}
& y=z+\delta w(z, \bar{z}, t), \\
& \bar{y}=\bar{z}+\delta \overline{w(z, \bar{z}, t)},
\end{aligned}
$$

which is parametrised by $t$. This transformation is consistent and invertible for points in a disk $\mathscr{D} \quad\{z \in \mathscr{D}:|z|<Z\}$-that is, there exist continuous functions $\zeta_{1}$ and $\zeta_{2}$ such that at any $t$

$$
z=y+\delta \zeta_{1}(y, \bar{y}), \quad \bar{z}=\bar{y}+\delta \zeta_{2}(y, \bar{y})
$$

and also

$$
\zeta_{1}(y, \bar{y})=\overline{\zeta_{2}(y, \bar{y})}
$$

The differential equation governing $z$ is therefore

$$
\begin{aligned}
\frac{d z}{d t}+ & \delta\left\{\frac{\partial w}{\partial z} \frac{d z}{d t}+\frac{\partial w}{\partial \bar{z}} \frac{d \bar{z}}{d t}\right\} \\
= & \delta\left\{f(z) C\left(\varepsilon^{1 / 2} t\right)+g(z+\delta w, \bar{z}+\delta \bar{w}, t)-g(z, \bar{z}, t)\right\} \\
& +\delta\{f(z+\delta w)-f(z)\} C\left(\varepsilon^{1 / 2} t\right)
\end{aligned}
$$

with initial condition

$$
z(0)=\alpha,
$$

and required also is $|\alpha|<Z$.

The presence of the term

$$
\delta \frac{\partial w}{\partial \bar{z}} \frac{d \bar{z}}{d t}
$$

in (4.14) complicates the issue slightly, but the validity proof given by Guckenheimer and Holmes [2] is usable after consideration of the system consisting of (4.14) and the equation governing the conjugate $\bar{z}(t)$ (obtained by taking the conjugate of (4.14) and noting that, since $t$ is a real variable, relations hold

and

$$
\frac{\overline{d z}}{d t}=\frac{d \bar{z}}{d t}
$$

$$
\overline{f(z)}=\bar{f}(\bar{z}) ; \quad \overline{w(z, \bar{z}, t)}=\bar{w}(\bar{z}, z, t) ; \quad \overline{g(z, \bar{z}, t)}=\bar{g}(\bar{z}, z, t)) .
$$

This last differential equation is made consistent with (4.14) by imposing the proper initial condition $\bar{z}(0)=\bar{\alpha}$. The new system (4.14 and its conjugate) has the form

$$
(\mathbf{I}+\delta \mathbf{W}) \frac{d}{d t}\left[\begin{array}{l}
z \\
\bar{z}
\end{array}\right]=\delta\left[\begin{array}{ll}
f(z) & C\left(\varepsilon^{1 / 2} t\right) \\
\bar{f}(\bar{z}) & \bar{C}\left(\varepsilon^{1 / 2} t\right)
\end{array}\right]+\delta^{2} \mathbf{G}(z, \bar{z}, t)
$$


where $I$ is the $2 \times 2$ identity matrix, the matrix $W$ is defined

$$
\mathbf{W}=\left[\begin{array}{ll}
\frac{\partial w}{\partial z} & \frac{\partial w}{\partial \bar{z}} \\
\frac{\partial \bar{w}}{\partial z} & \frac{\partial \bar{w}}{\partial \bar{z}}
\end{array}\right]
$$

and the entries $G_{j}$ in the complex 2 -vector $\mathbf{G}$ are uniformly bounded in modulus

by the bound inequality (4.4).

$$
\left|G_{j}\right| \leq\left(\mathscr{K}^{2}+\mathscr{K}^{3}\right)
$$

The matrix $(\mathbf{I}+\delta \mathbf{W})$ is invertible and its inverse can be expanded as a power series for sufficiently small $\delta$. So the proof given by Guckenheimer and Holmes [2] carries through, interpreted where necessary in complex variable formalism. In using their error bound inequality (4.1.10) in [2], the initial value of the error is zero, and the integral in that formula is assigned its value, rather than a bound, so that for the present use the error growth is

$$
\left|z(t)-z_{0}(t)\right| \leq \delta \mathscr{K}(1+2 \mathscr{K})(\exp (\delta \mathscr{K} t)-1) .
$$

(Here (4.18) has been used, and $\mathscr{K}$ replaces the Lipschitz constant $L$ used in [2].)

The implications of this last result are the following. On a $|t|$ interval of $O\left(\delta^{-1}|\ln \delta|\right)$, solution existence is assured, that is to say, both

$$
|z(t)|,\left|z_{0}(t)\right|<Z \text {. }
$$

Further, on any $|t|$ interval such that the product $(\delta \mathscr{K} t)$ is small, the bound on the error growth rate (4.19) is approximately linear

$$
\left|z(t)-z_{0}(t)\right| \leq \delta^{2} \mathscr{K}^{2}(1+2 \mathscr{K}) t=\varepsilon^{2 Q+1} \mathscr{K}^{2}(1+2 \mathscr{K}) t .
$$

Thus the approximate solution $z_{0}(t)(4.11)$ is defensible only on intervals at most $|t| \sim O\left(\varepsilon^{-\gamma}\right)$ with $-\gamma+2 Q+1=Q$, that is,

$$
\gamma=Q+1 \text {. }
$$

In the problem discussed in Sections 2 and 3 above, the parameter $Q=1 / 2$ so that an upper bound on $\gamma$ based on the calculation (4.21) is $3 / 2$, which is clearly large enough to allow $C$ to relax sufficiently to be satisfactorily approximated asymptotically within an acceptable interval of $|t|$. (In fact $\gamma$ is limited by the fundamental existence interval $|t| \sim O\left(\delta^{-1}|\ln \delta|\right) \sim$ $O\left(\varepsilon^{-Q-1 / 2}|\ln \varepsilon|\right)$, so the approximation $z_{0}$ is a good one on any shorter interval). Thus the analysis of those sections-in particular the matching with outer solutions-is justified.

\section{Discussion of previous studies}

In a study [5] Kevorkian claims to be able to identify by an approximate theory a "distinguished solution" of equations inessentially different from 
(2.2-5) which leads to sustained resonance. This solution is one associated with a trajectory passing through a saddle point in a phase-plane diagram generated by an equation locally modelling the dynamics in the $t$ interval on which $p$ is close to $\omega$, and the solution is claimed to be available for all values of the small parameter. The resonance is an unusual one, both in occurrence and character; the erroneous conclusion drawn from the analysis is a consequence of the cited author's failure to establish uniform applicability of his inner solution on a $t$-interval which is at least $O\left(\varepsilon^{-1 / 2}\right)$. This contention is also addressed in the following.

Empirically, the distinguished solution of Kevorkian's central result describes an unusual dynamic instability, although it is certainly a feature of the equations. As was demonstrated above in Section 2, it predicts a (slow) divergence in spin $p$ (angular velocity about the symmetry axis of the projectile) and a concommitant increase in frequency of the pitch/yaw $(x)$ oscillation (to a fixed observer, rate of precession of the symmetry axis); the amplitude $\rho$ ([5], equation $4.10 \mathrm{a}$ ) of the latter is locally almost unaffected, although it slowly decays-(2.19) above shows that

$$
x \simeq A \omega^{-1 / 2} \cos (2(\omega-1) / \varepsilon+\psi(0)) .
$$

The increase in the total rotational energy of the projectile which, on the basis of the present (2.19) is approximately

$$
p^{2}+(\omega x)^{2}+(d x / d t)^{2} \sim \omega^{2}+A^{2} \omega
$$

might conceivably be extracted from its translational kinetic energy by aerodynamic coupling. Even if the basic pitch/yaw amplitude $A$ were large, such a divergence might not be an exceptional concern: the system is simply recovering from a significant disturbance. (Anecdotal evidence of observations of such recovery is known to the writer.)

However, readers familiar with the behaviour of spun projectiles in the atmosphere will be more accustomed to perturbation-induced divergence from spin about the symmetry axis to gyration about an axis roughly tangent to the original trajectory, and in a plane perpendicular to the symmetry axis of the projectile. (The apparently angular-momentum-conserving, perturbationinduced gyration of the "underspun", high-velocity small-arms projectile is suggested.) Here the energy demands of the transfer are essentially supplied by the initial spin energy, although possibly augmented by aerodynamic couples drawing on the translational kinetic energy if angular momentum is not conserved. Although the situation modelled here is not the same, it would be unremarkable to find similarities in the basic dynamics. 
Error estimates for Kervorkian's study [5] can be made by recapturing his results using methods for which such estimates can be inferred. It transpires that Kevorkian's description of the dynamics ([5], Section 4) also follows from use of the averaging algorithm in a slightly non-standard context. To see this, define

$$
\begin{aligned}
p & =1+\varepsilon^{1 / 2} p_{1}, \\
p_{1}(0) & =0, \\
\hat{\theta} & =\psi-t-\psi(0), \\
\hat{\theta}(0) & =0
\end{aligned}
$$

and write (2.2-4) as the system

$$
\begin{aligned}
& \frac{d x}{d t}=y, \\
& \frac{d y}{d t}=-\frac{1}{2}\left(1+\omega^{2}\right) x-\left(\varepsilon^{1 / 2} p-1+\left\{\varepsilon p_{1}^{2} / 2\right\}\right) x, \\
& \frac{d p_{1}}{d t}=\varepsilon^{1 / 2} x \sin (\hat{\theta}+t+\psi(0)), \\
& \frac{d \hat{\theta}}{d t}=\varepsilon^{1 / 2} p_{1} .
\end{aligned}
$$

To study the dynamics of the resonant interaction, Kevorkian ([5], equation 4.7) truncates the power series expansion of the exponential (see 2.5 above)

$$
\omega^{2}=1+\varepsilon t
$$

and, in effect and reasonably, omits the \{\} bracketed, second-order term in (5.6) so that it becomes

$$
\begin{aligned}
\frac{d y}{d t} & =-x-\varepsilon^{1 / 2}\left(p_{1}+\varepsilon^{1 / 2} t\right) x \\
& \equiv-x-\varepsilon^{1 / 2} w x
\end{aligned}
$$

where the new variable $w$ is defined by implication, and has the property

$$
w(0)=0 .
$$

To present the system $(5.5,5.10,5.7-8)$ in averagable form, introduce new variables

$$
\begin{aligned}
& x=r \cos (t)+s \sin (t), \\
& y=-r \sin (t)+s \cos (t)
\end{aligned}
$$


and on replacing $p_{1}$ by $w$ as a variable, obtain the system

$$
\begin{aligned}
& \frac{d r}{d t}=\varepsilon^{1 / 2} w(r \cos (t)+s \sin (t)) \sin (t), \\
& \frac{d s}{d t}=-\varepsilon^{1 / 2} w(r \cos (t)+s \sin (t)) \sin (t), \\
& \frac{d w}{d t}=\varepsilon^{1 / 2}[(r \cos (t)+\dot{s} \sin (t)) \sin (\hat{\theta}+t+\psi(0))+1], \\
& \frac{d \hat{\theta}}{d t}=\varepsilon^{1 / 2}\left(w-\varepsilon^{1 / 2} t\right) .
\end{aligned}
$$

The system (5.14-17) almost satisfies the conditions for application of the averaging theorem (see, for example [2]). If the $(\varepsilon t)$ were dropped from (5.17) it would do so, and in that case the solution vector of the truncated system would be approximated as

$$
\left[\begin{array}{c}
r \\
s \\
w \\
\hat{\theta}
\end{array}\right]=\left[\begin{array}{c}
r(0) \\
s(0) \\
w(0) \\
\hat{\theta}(0)
\end{array}\right]+\varepsilon^{1 / 2}\left[\begin{array}{c}
\rho \\
\sigma \\
\Omega \\
\mu
\end{array}\right]+\mathbf{E}
$$

on an interval $|t| \leq k \varepsilon^{-1}$, with $a$ an error $\mathbf{E}$ growth bound which is in effect

$$
\|\mathbf{E}(t)\| \leq L \varepsilon T
$$

for $|t|$ of $O\left(\varepsilon^{-1 / 2}\right)$. (This last statement is inferred if the inequality 4.1.10 in [2] is approximated for non-large values of the argument of its exponential when, as in Section 4 above, the integral in that inequality is evaluated rather than bounded. Note that in the present discussion, the small parameter is $\varepsilon^{1 / 2}$ but in [2] it is $\varepsilon$.) Here $k$ and $L$ are two $O(1)$ constraints, and the explicit parts of the approximation $(\rho, \sigma, \Omega, \mu)$, which are governed by equations deducible from those developed below, also have a unit order bound on the interval.

It is immediately clear that error growth bound (5.19) is such that the explicit, non-constant terms in the approximation (5.18) can only be guaranteed to be distinguishable from its error on a $|t|$ interval that is small in comparison with $\varepsilon^{-1 / 2}$. This limitation is crucial to any inferences to be made from the theory. For Kevorkian's purpose-that is, a matching with "outer" solutions developed for $|t|$ much greater than $\varepsilon^{-1 / 2}$-this validity interval would be inadequate.

We next show how Kevorkian's ([5], Section 4) description of the resonant interaction can be extracted by averaging (5.14-16), and keeping (5.17) as it stands. In this case it is possible to prove results essentially identical to those (5.18-19) above obtained when $(\varepsilon t)$ is omitted by using a straightforward 
modification of the proof shown in [2]. In particular, the error growth bound (5.19) remains effectively unaltered.

Equations (5.14-16) can be re-expressed as

$$
\begin{aligned}
\frac{d r}{d t} & =\frac{1}{2} \varepsilon^{1 / 2} w s+\frac{1}{2} \varepsilon^{1 / 2} w(r \sin (2 t)-s \sin (2 t)) \\
\frac{d s}{d t} & =-\frac{1}{2} \varepsilon^{1 / 2} w r-\frac{1}{2} \varepsilon^{1 / 2} w(s \sin (2 t)+r \sin (2 t)) \\
\frac{d w}{d t} & =\frac{1}{2} \varepsilon^{1 / 2}(r \sin (\hat{\theta}+\psi(0))+s \cos (\hat{\theta}+\psi(0))+2) \\
& +\frac{1}{2} \varepsilon^{1 / 2}(r \cos (2 t) \sin (\hat{\theta}+\psi(0))-s \sin (2 t) \cos (\hat{\theta}+\psi(0)))
\end{aligned}
$$

so the equations governing the explicit part of the averaged approximation are

$$
\begin{aligned}
\frac{d r}{d t} & =\frac{1}{2} \varepsilon^{1 / 2} s w, \\
\frac{d s}{d t} & =-\frac{1}{2} \varepsilon^{1 / 2} r w, \\
\frac{d w}{d t} & =\frac{1}{2} \varepsilon^{1 / 2}(r \sin (\hat{\theta}+\psi(0))+s \cos (\hat{\theta}+\psi(0))+2) .
\end{aligned}
$$

The first two (5.23-24) of these equations have a closed form solution

$$
\begin{aligned}
& r=\rho_{0} \sin \left(\varepsilon^{1 / 2} \frac{\Theta}{2}+\beta\right), \\
& s=\rho_{0} \cos \left(\varepsilon^{1 / 2} \frac{\Theta}{2}+\beta\right)
\end{aligned}
$$

in terms of a new variable

$$
\begin{aligned}
\varepsilon^{1 / 2} \Theta & =\varepsilon^{1 / 2} \int_{0}^{t} w d t \\
& =\varepsilon^{1 / 2} \int_{0}^{t} p_{1} d t+\frac{1}{2} \varepsilon t^{2} \\
& \equiv \hat{\theta}+\frac{1}{2} \varepsilon t^{2}
\end{aligned}
$$

and constants $\rho_{0}$ and $\beta$ chosen to satisfy initial conditions $r(0)$ and $s(0)$. Two remarks are apposite here. In the first place these solutions are equivalent to Kevorkian's [5] (4.9a,b with 4.10a,b); and in the second, their closed form produces a seductive but illusory impression of uniform applicability. This last contention follows from the averaging theorem, and the error growth bound quoted above (inequality 5.19). It is less misleading to state 
these results $(5.26-27)$ in the form (see 5.18)

$$
\begin{aligned}
& r=\rho_{0} \sin (\beta)+\rho_{0} \varepsilon^{1 / 2} \frac{\Theta}{2} \cos (\beta)+E_{r}, \\
& s=\rho_{0} \cos (\beta)-\rho_{0} \varepsilon^{1 / 2} \frac{\Theta}{2} \sin (\beta)+E_{s}
\end{aligned}
$$

for $t \sim O\left(\varepsilon^{q-1 / 2}\right)$ and any $q>0$, where the errors $E_{r}$ and $E_{s}$ are $\sim$ $O\left(\varepsilon^{q+1 / 2}\right)$.

To complete the reproduction of Kevorkian's description, substitute (5.2627) into (5.25) and use (5.28) to obtain an equation

$$
\frac{d w}{d t}=\frac{1}{2} \varepsilon^{1 / 2} \rho_{0}\left(\cos \left(\frac{\hat{\theta}}{2}-\frac{\varepsilon t^{2}}{4}+\psi(0)-\beta\right)+2 / \rho_{0}\right)
$$

which can be re-expressed using (5.17) as

$$
\frac{d^{2} \hat{\theta}}{d t^{2}}=\frac{1}{2} \varepsilon \rho_{0}\left(\cos \left(\frac{\hat{\theta}}{2}-\frac{\varepsilon t^{2}}{4}+\psi(0)-\beta\right)\right) .
$$

The introduction of a slow variable $\bar{t}=\varepsilon^{1 / 2} t$ and a redefinition $\theta=\hat{\theta}-\frac{e t^{2}}{2}$ makes (5.31) equivalent to Kevorkian's [5] (4.10c), which is central to his local theory. Thus, as foreshadowed, Kevorkian's description of the resonant interaction is an application of averaging, and is subject to the limitations of that theory as set out above. It cannot therefore be used to make predictions concerning the evolution of the system.

In contrast, the application of averaging to the problem in the present study is, in a sense, an indirect one. The preliminary transformation (3.67) ensures that the small coefficient in (3.30) which is averaged is $\varepsilon$ rather than $\varepsilon^{1 / 2}$, while the independent variable remains a perturbation of $t$. Thus averaging gives a uniform description of the process on a $t$ scale which is large enough to allow matching with the outer solutions. The availability of these solutions on the extended interval, different from those of Kervorkianthey show neither the sustained resonant solution, nor its neighbours-means that extrapolation of the Kevorkian solutions is an invalid procedure.

Kath [3], [4] recognises the shortcoming of the Kevorkian approachsee the remark following equation 2.14 in [3], which notes the necessity of an $O\left(\varepsilon^{-1}\right)$ existence interval for approximate solutions in the transient resonance. He attempts to calculate the resonant interaction directly, if not explicitly. However, his computation is not internally consistent. In the reduction in [3] which takes the first of those equations (2.12) to the first of equations (2.14) (via the substitution (2.13)) it is assumed that $(p \approx) P \approx \omega$ on what is required to be an $O\left(\varepsilon^{-1}\right) t$ interval. This ansatz carries through, 
and is re-used in the averaged form

$$
\left\langle\frac{d \lambda}{d \tau}\right\rangle=\mu^{-1}\left\langle\frac{d(P-\omega)}{d \tau}\right\rangle=0,
$$

in the calculation of $\langle\sin (\theta)\rangle$. (See the second of equations 2.14 and equation 3.12 in [3].) The calculations which follow establish what is in effect a multiplicative, supplementary $x$ amplitude growth equation (3.11 in [3]) whose solution (3.13) fixes that growth rate as proportional to $\omega^{1 / 8}$. Irrespective of the form of any monotone function $\omega \in C^{3}$, if both $(p-\omega)$ and its averaged $\tau$ derivative are small, application of the Liouville-Green approximation to equation (1.1) in [3] with $p \approx \omega$ is justified, and it will give an overall amplitude decay rate proportional to $\omega^{-1 / 2}$, rather than $\omega^{-3 / 8}$ as implied in [3] by equations (3.13), (2.1). An internally consistent approximate theory from the ansatz $p \approx \omega$ on an $O\left(\varepsilon^{-1}\right) t$ scale is given in Section 2 above, and it is shown there (2.27) that the additive perturbation correction to $p$ is proportional to $\omega^{3 / 8}$.

\section{Conclusions}

The description of the non-linear oscillator's resonant transition given in Section 3 above is fundamentally different from the one advanced in [5]. In physical terms, the present mechanism is best thought of as an energy exchange process between two modes (spin and pitch/yaw), and it is capable of proceeding in either direction, depending essentially on the value of a relative phase

$$
\arg (y(0))-\psi(0)
$$

This last cannot reasonably be assessed from initial conditions at $-\infty$, but for half the possible range of its values the energy flow is one way, and for the other half the flow is reversed. It is speculated that the pitch/yaw amplitudes, in cases when the energy flow is towards that mode, could become sufficiently large as to require unmodelled aerodynamic inputs which in turn lead to divergence.

Insofar as sustained resonance is concerned, the present description finds none for sufficiently small values of the small parameter $\varepsilon$ and given finite initial disturbance $\left|y_{-}\right|$. In contrast, the solution claimed in [5] predicts divergence to be possible no matter how small the $\varepsilon$ value, provided the product of the base spin and initial pitch/yaw amplitude is large enough. The sustained resonant solution can be identified (Section 2) but it is not available for the specified initial conditions. The analysis in Section 3 suggests that a measure of disturbance size at which that solution might become available is 
when

$$
Q=\varepsilon_{N}^{1 / 2} \hat{x}_{N}=2^{-1 / 4} \varepsilon^{1 / 2} K^{1 / 2} \hat{x} \sim 1
$$

Here the $N$ suffix on the scaled variables defined in (2.1) is reinstated, $\hat{x}$ denotes the initial dispersion magnitude in pitch/yaw ( $\alpha$ in Section 3), and $K$, strictly the spin at resonance, could be taken to be the initial spin rate. This contrasts the comparable result claimed in [5] that is, in the present notation, sustained resonance possible for $K \hat{x}>1$.

Finally, the result (3.29) (or 3.41) suggests a somewhat counter-intuitive method of reducing the response to the transient resonance. In terms of the original variables (that is, before introduction of the scalings (2.1)) the magnitude of the product $(\varepsilon K)^{1 / 2}$ is controlling, for given $|y(0)|$, so it suggests that reduction of the datum spin rate $K$ would reduce sensitivity to initial axial misalignment. After some thought it seemed to the writer that this idea was not without merit as:

1. The less the value of $K$, the higher the altitude at which resonance occurs, so the less the atmospheric density, and hence the less the aerodynamic coupling moment; and

2. The less the value of $K$, the less the spin energy available to be fed into the undesirable pitch/yaw mode. (For the connection between the time variable and altitude refer Kevorkian [4].) But the reduction of $K$ should not be sufficient to make the problem a perturbation from some $p=0$ analysis which, as suggested in Section 2, may present its own stability problem.

\section{References}

[1] P. B. Chapman, "A uniform description of an oscillator's resonant transition," J. Aust. Math. Soc. (B) 32 (1990) 193-206.

[2] J. Guckenheimer and P. Holmes, Nonlinear oscillations, dynamical systems, and bifurcations of vector fields. (Springer-Verlag, N.Y., 1983).

[3] W. L. Kath, "Necessary conditions for sustained resonance." SIAM J. Appl. Math. 43 (1983) 314-322.

[4] W. L. Kath, "Conditions for sustained resonance." SIAM J. Appl. Math. 43 (1983) 579583.

[5] J. Kevorkian, “On a model for re-entry roll resonance." SIAM J. Appl. Math. 26 (1974) 638-669.

[6] J. Kevorkian, "Perturbation techniques for oscillatory systems with slowly varying coefficients." SIAM Review 29 (1987) 391-461.

[7] M. J. Lighthill, "A technique for rendering approximate solutions to physical problems uniformly valid." Philos. Mag. 7, 40 (1949) 1179-1201.

[8] F. W. J. Olver, Asymptotics and special functions. (Academic Press, N.Y., 1974)

[9] G. N. Watson, Theory of Bessel functions. Second edition. (C.U.P., London, 1962). 